IP/BBSR/2000-18

hep-th/0005113

\title{
Noncommutative Open String, D-brane and Duality
}

\author{
Jnanadeva Maharana and Shesansu S. Pal \\ e-mail:maharana@iopb.res.in and shesansu@iopb.res.in \\ Institute of Physics \\ Bhubaneswar - 751005, India
}

\begin{abstract}
We consider open strings ending on D-branes in the presence of constant metric, G, antisymmetric tensor, B and gauge field, A. The Hamiltonian is manifestly invariant under a global noncompact group; strikingly similar to toroidally compactified closed string Hamiltonian. The evolution equations for the string coordinates, $\left\{X^{i}\right\}$ and their dual partners, $\left\{Y_{i}\right\}$, are combined to obtain equations of motion invariant under the noncompact symmetry transformations. We show that one can start from a noncommutative theory, with nonvanishing $\mathrm{G}$ and $\mathrm{B}$ and mixed boundary conditions and then go over to a dual theory whose coordinates obey Dirichlet boundary conditions. It is possible to generate B-field by implementing the noncompact symmetry transformation. The connection between this duality transformation and Seiberg-Witten map is discussed.
\end{abstract}


The discovery of noncommutativity property in string theory and in field theories has attracted considerable attention in recent times. When one compactifies the $\mathrm{M}$ (atrix) model on a $T^{2}$ in the presence of antisymmetric tensor, noncommutative supersymmetric Yang-Mills theory appear naturally as was shown by Connes, Douglas and Schwarz [1]. This can be understood from the D-string point of view if one dualizes one cycle of the torus [2]. Subsequently, the origin of the noncommutativity property from the perspective of D0-branes have been studied by several authors [3, 4]. Another interesting result is that the D-brane worldvolume exhibits noncommutativity in string theory even before one goes over to the $\mathrm{M}$ (atrix) model limit [5, 6]. Furthermore, there have been attempts to explain noncommutativity on D-brane world volume through the study of open string quantization in the presence of background fields [7, 8, 9, 10]. Recently, Seiberg and Witten [11 have provided deeper insight into the relation between string theory and noncommutative geometry and they have investigated various aspects of nonabelian gauge theories in this context.

Recent progress in string theory has enriched our understanding of the dynamics of string theory and has revealed interconnection between the five perturbatively distinct theories [12]. It is recognized that dualities have played a cardinal role in these developments [13, 14, 15]. The discovery of Dp-branes has opened up investigations in new directions in string theories [16] and we can visualize these objects as spacetime hypersurfaces on which open strings can end. Consequently, one can explore various aspects of string dynamics and establish connections between string theory and supersymmetric Yang-Mills theories from a novel perspective.It has been conjectured that strongly coupled spatially noncommutative $\mathcal{N}=4 \mathrm{SYM}$ has a dual description in terms of open string theory in a near critical electric field [17]. Some aspects of T-duality, in the context of Morita equivalence, have been studied [18, 11] and the T-duality properties of open strings in the presence of B-field have been considered from the point of view of canonical transformations [19. However, as we shall see below, we utilize duality transformation which is analog of $O(d, d)$ transformation and such transformations have interesting consequences. It is natural to expect that the brane world might reveal interesting attributes when we examine the properties of its spacetime geometry. Where one quantizes an open string ending on a D-brane with a constant antisymmetric field background (coming from NS-NS sector), then it can be shown that spacetime coordinates of the open string endpoints are noncommutative. The purpose of this investigation is to explore the consequences of target space duality for the configurations when open strings end on D-branes and there is constant background metric, $G_{i j}$ and NS-NS antisymmetric tensor field, $B_{i j}$. We intend to explore a scenario where one starts from a theory with noncommuting string coordinates and then go over to a dual set of coordinates and backgrounds and examine whether the dual theory is a noncommutative or commutative one. We shall also consider the situation when the two end points of the open string are attached to the same brane and we couple string to the resulting $U(1)$ gauge field with constant field 
strength, i.e. $F=d A$ is constant. We show that the Hamiltonian obtained from the worldsheet action can be cast in a form similar to the one derived for closed strings for constant backgrounds exhibiting $O(d, d)$ invariance. We argue that there is an analogue of the $O(d, d)$ symmetry in this situation. It is well known that if one has an open string where some of the coordinates obey the Dirichlet boundary conditions and the rest of them satisfy Neumann boundary conditions, then the usual T-duality operation interchanges these boundary conditions to one another. We also study how the boundary conditions are modified under the duality transformations mentioned above.

Let us consider, for the sake of definiteness, a fundamental bosonic string ending on a Dp-brane, although our considerations are applicable to type II superstring or type 0 superstring. The action [20, 21], in the orthonormal gauge, is given by

$$
S=-\frac{1}{2} \int d^{2} \sigma\left(\gamma^{\alpha \beta} G_{i j} \partial_{\alpha} X^{i} \partial_{\beta} X^{j}+\epsilon^{\alpha \beta} \partial_{\alpha} X^{i} \partial_{\beta} X^{j} B_{i j}\right)-\int_{\partial \Sigma} d \tau A_{i} \partial_{\tau} X^{i}
$$

where $\gamma^{\alpha \beta}=\operatorname{diag}(-11), \epsilon^{01}=1, \epsilon^{10}=-1$ and $\left\{X^{i}\right\}, i=0,1 \ldots p$ are the coordinates of the string along the brane directions. The constant nonvanishing backgrounds are: metric, $G_{i j}$ and antisymmetric tensor, $B_{i j}, i, j=0,1 . . p$, with $\mathrm{H}=\mathrm{dB}=0$, The rest of the coordinates are denoted as $X^{a}, a=p+1, . . D$ and we have not written the action for these coordinates here. Moreover, we envisage the configuration when the two ends of the open string are attached to the same Dp-brane and $A_{i}$ is the resulting $\mathrm{U}(1)$ gauge field living on the world volume of the brane. The last term in eq.(11) is the coupling of the gauge connection to the string. We could rewrite this piece as $\frac{1}{2} \int_{\Sigma} d^{2} \sigma \epsilon^{\alpha \beta} F_{i j} \partial_{\alpha} X^{i} \partial_{\beta} X^{j}$, where field strength, $F_{i j}$ is taken as constant. As a consequence, (11) can be expressed as

$$
S=-\frac{1}{2} \int d^{2} \sigma\left[\gamma^{\alpha \beta} G_{i j} \partial_{\alpha} X^{i} \partial_{\beta} X^{j}+\epsilon^{\alpha \beta} \partial_{\alpha} X^{i} \partial_{\beta} X^{j} B_{i j}-\epsilon^{\alpha \beta} F_{i j} \partial_{\alpha} X^{i} \partial_{\beta} X^{j}\right]
$$

The resulting equation of motion is

$$
\partial_{\alpha}\left(\gamma^{\alpha \beta} G_{i j} \partial_{\beta} X^{j}-\epsilon^{\alpha \beta}\left[F_{i j}-B_{i j}\right] \partial_{\beta} X^{j}\right)=0
$$

the boundary conditions at $\sigma=0, \pi$ are

$$
G_{i j} \partial_{\sigma} X^{j}-\mathcal{F}_{i j} \partial_{\tau} X^{j}=0
$$

where $\mathcal{F}_{i j}=B_{i j}-F_{i j}$. The noncommutativity character of the string coordinates is revealed as follows. First the worldsheet coordinates are complexified through the definition $z=\tau+i \sigma$, after $\tau$ has been rotated to Euclidean signature. Next, one maps the disc geometry of the openstring to the upper half z-plane and then computes the correlation function $\left\langle X^{i}(z) X^{j}\left(z^{\prime}\right)>\right.$ with the above boundary condition (舟), which has the following form. [11]

$$
\left\langle X^{i}(z) X^{j}\left(z^{\prime}\right)\right\rangle=-\frac{1}{2 \pi}\left[G^{i j} \log \left|z-z^{\prime}\right|-G^{i j} \log \left|z-\bar{z}^{\prime}\right|+\bar{G}^{i j} \log \left|z-\bar{z}^{\prime}\right|^{2}+\theta^{i j} \log \frac{z-\bar{z}^{\prime}}{\bar{z}-z^{\prime}}\right]
$$


Where $\bar{G}^{i j}=\left[(G+\mathcal{F})^{-1} G(G-\mathcal{F})^{-1}\right]^{i j}$ and $\theta^{i j}=-\left[(G+\mathcal{F})^{-1} \mathcal{F}(G-\mathcal{F})^{-1}\right]^{i j}$ The above result can be derived in a straight forward manner following the work of Abouelsaood et al [22]. It is of interest to consider the propagator on the boundary which has the disc geometry

$$
<X^{i}(\tau) X^{j}\left(\tau^{\prime}\right)>=-\frac{1}{2 \pi} \bar{G}^{i j} \log \left(\tau-\tau^{\prime}\right)^{2}+\frac{i}{2} \theta^{i j} \epsilon\left(\tau-\tau^{\prime}\right)
$$

where $\epsilon(\tau)$ takes values +1 and -1 for positive and negative argument respectively. Note that $\bar{G}^{i j}$ is the effective metric seen by the open string. Moreover, this also determines the anomalous dimension of the vertex operator as this metric appears as the coefficient of the $\log \left(\tau-\tau^{\prime}\right)^{2}$. Note that the noncommutativity property of the spatial coordinates can be brought out starting from the correlation functions of the string coordinates and then defining them on the real z-axis. The noncommutativity feature of the coordinates $X^{i}$ is derived from the structure of the correlation function.

However, at this stage, we proceed to derive the Hamiltonian associated with the action (2), keeping Lorentzian signature for $(\tau, \sigma)$. The canonical momenta are given by

$$
P_{i}=G_{i j} \dot{X}^{j}-\mathcal{F}_{i j} X^{\prime j}
$$

note that dot and prime refer to the derivatives with respect to $\tau$ and $\sigma$ here and everywhere. The Hamiltonian density is given by

$$
H=\frac{1}{2}\left(\begin{array}{cc}
P_{i} & X^{\prime i}
\end{array}\right) \mathcal{M}_{i j}\left(\begin{array}{c}
P_{j} \\
X^{\prime j}
\end{array}\right)
$$

where

$$
\mathcal{M}_{i j}=\left(\begin{array}{cc}
G^{i j} & G^{i k} \mathcal{F}_{k j} \\
-\mathcal{F}_{i k} G^{k j} & G_{i j}-\mathcal{F}_{i k} G^{k l} \mathcal{F}_{l j}
\end{array}\right)
$$

Notice the form of the Hamiltonian with $\mathcal{M}$-matrix. This has striking similarity with the expression one derives for the Hamiltonian that appeared for closed string with constant background metric and antisymmetric field $\left(B_{i j}\right)$ where the corresponding M-matrix appears with specific combinations of the backgrounds 23]. In the present context, we note the appearance of $\mathcal{F}$ in the the $\mathcal{M}$-matrix which has replaced the -B-field of the M-matrix. We may argue that with the appearance of symmetric $\mathcal{M}$-matrix and the combination of $P_{i}$ and $X^{\prime i}$ in the expression (8) implies that the Hamiltonian is $O(p+1, p+1)$ invariant; if we demand the transformations

$$
\left(\begin{array}{c}
P_{i} \\
X^{\prime i}
\end{array}\right) \rightarrow\left(\Omega^{-1}\right)_{i j}\left(\begin{array}{c}
P_{j} \\
X^{\prime j}
\end{array}\right) \quad \mathcal{M} \rightarrow \Omega^{T} \mathcal{M} \Omega \quad \text { and } \quad \Omega \eta \Omega^{T}=\eta
$$

Here $\eta=\left(\begin{array}{ll}0 & \mathbf{1} \\ \mathbf{1} & 0\end{array}\right)$ is the metric of the $O(p+1, p+1)$ group, $\mathbf{1}$ being $(p+1) \times(p+1)$ unit matrix. $\Omega$ is, $(2 p+2) \times(2 p+2)$ matrix, which is an arbitrary element of the the 
global $O(p+1, p+1)$ group. Let us set the gauge potential $A_{i}=0$ from now on, for the sake of simplicity.

A few comments are in order at this stage. In the case of toroidal compactification, when d-spatial coordinates are compactified on $T^{d}$ [24] the T-duality group is $O(d, d, Z)$ and this is a generalization of the $R \rightarrow \frac{1}{R}$ duality appearing in the case of compactification on $S^{1}$. As is well known, if we consider a theory with a given set of background fields which is described by a conformal field theory, these duality transformations can take us to another set of background fields and we can have a conformal field theory for the new set.

When we consider open string theories with some of the coordinates obeying Neumann boundary condition and the rest of the set fulfilling Dirichlet boundary conditions, the simplest form of T-duality (i.e. analogue $R \rightarrow \frac{1}{R}$ transformation) interchanges the two types of boundary conditions (actually it is $P \leftrightarrow X^{\prime}$ transformation for trivial backgrounds). Therefore, in view of the appearance of afore mentioned $O(p+1, p+1)$ symmetry, we expect that starting from a constant $G_{i j}$ we can generate both $\mathrm{G}$ and $\mathrm{B}$ with a judicious choice of $\Omega$ matrix. Thus, one might consider, to begin with, background configurations with constant $G_{i j}$ and $B_{i j}$ and choose the boundary condition $G_{i j} X^{\prime j}-B_{i j} \dot{X}^{j}=0$, at $\sigma=0, \pi$. In this case,correlation functions of the string coordinates $X^{i}(z)$ and $X^{j}\left(z^{\prime}\right)$ are given by (5). Then by going over to a dual theory, we shall find that the coordinates of that theory are commutative.

In order to proceed in this direction we need to consider the underlying T-duality symmetry while studying evolution equations of the worldsheet coordinates. This is revealed in an elegant manner [25, 26, 27] by introducing auxiliary fields, $U_{\alpha}^{i}$, described below. Let us consider the Lagrangian density

$$
\mathcal{L}_{1}=-\frac{1}{2} \gamma^{\alpha \beta} U_{\alpha}^{i} U_{\beta}^{j} G_{i j}-\frac{1}{2} \epsilon^{\alpha \beta} U_{\alpha}^{i} U_{\beta}^{j} B_{i j}+\partial_{\alpha} X^{i}\left(\gamma^{\alpha \beta} U_{\beta}^{j} G_{i j}+\epsilon^{\alpha \beta} U_{\beta}^{j} B_{i j}\right)
$$

The equations of motion associated with $U_{\beta}^{j}$ and $X^{i}$, respectively, are

$$
\begin{gathered}
\left(\partial_{\alpha} X^{i}-U_{\alpha}^{i}\right)\left(\gamma^{\alpha \beta} G_{i j}+\epsilon^{\alpha \beta} B_{i j}\right)=0 \\
\partial_{\alpha}\left(\gamma^{\alpha \beta} U_{\beta}^{j} G_{i j}+\epsilon^{\alpha \beta} U_{\beta}^{j} B_{i j}\right)=0
\end{gathered}
$$

If we solve for $U_{\alpha}^{i}$ from (12) and substitute in (13) we recover the equation of motion for $\left\{X^{i}\right\}$ coordinates at the classical level. Now let us introduce a set of 'dual' coordinates $\left\{Y_{i}\right\}$ and correspondingly auxiliary fields $\left\{V_{\alpha}^{i}\right\}$ and another Lagrangian density

$$
\mathcal{L}_{2}=\frac{1}{2} \gamma^{\alpha \beta} V_{\alpha}^{i} V_{\beta}^{j} G_{i j}+\frac{1}{2} \epsilon^{\alpha \beta} V_{\alpha}^{i} V_{\beta}^{j} B_{i j}+\epsilon^{\alpha \beta} \partial_{\alpha} Y_{i} V_{\beta}^{i}
$$

The equations of motion associated with $V_{\alpha}^{i}$ and $Y_{i}$ are

$$
\gamma^{\alpha \beta} V_{\beta}^{j} G_{i j}+\epsilon^{\alpha \beta} V_{\beta}^{j} B_{i j}+\epsilon^{\beta \alpha} \partial_{\beta} Y_{i}=0
$$




$$
\partial_{\alpha}\left(\epsilon^{\alpha \beta} V_{\beta}^{i}\right)=0
$$

We can express $V_{\alpha}^{i}$ in terms of $Y_{i}$ coordinates through the relation

$$
V_{\alpha}^{i}=\gamma_{\alpha \beta} \epsilon^{\beta \delta} P^{i j} \partial_{\delta} Y_{j}+Q^{i j} \partial_{\alpha} Y_{j}
$$

where

$$
P=B^{-1}\left(G B^{-1}-B G^{-1}\right)^{-1} \quad \text { and } \quad Q=-G^{-1}\left(G B^{-1}-B G^{-1}\right)^{-1}
$$

are symmetric and antisymmetric tensors respectively.

The equations of motion derived from (11) suggest that we can write $U_{i}^{\alpha}=\epsilon^{\alpha \beta} \partial_{\beta} Y_{i}$, at least locally, and similarly we may conclude, from equations of motion, associated with $\mathcal{L}_{2}$, for $Y_{i}$ coordinates that

$$
\frac{\partial \mathcal{L}_{2}}{\partial\left(\partial_{\alpha} Y_{i}\right)}=\epsilon^{\alpha \beta} \partial_{\beta} X^{i}
$$

Therefore, we have two sets of local relations

$$
\begin{gathered}
\epsilon^{\alpha \beta} \partial_{\beta} Y_{i}=\gamma^{\alpha \beta} \partial_{\beta} X^{j} G_{i j}+\epsilon^{\alpha \beta} \partial_{\beta} X^{j} B_{i j} \\
\epsilon^{\alpha \beta} \partial_{\beta} X^{i}=\gamma^{\alpha \beta} \partial_{\beta} Y_{i} P^{i j}+\epsilon^{\alpha \beta} \partial_{\beta} Y_{j} Q^{i j}
\end{gathered}
$$

If we examine the field equations for $\left\{X^{i}\right\}$ coordinates, it is the divergence of the r.h.s of (20). But this is nothing but the Bianchi identity for the dual coordinates $Y_{i}$. The same is true when we consider (21). This is all very familiar whenever we consider such dualities; the field equations for one set is the Bianchi identity for the dual variables and vice versa. An important point deserves to be mentioned en passant: these two evolution equations can be expressed in a simple and elegant form [25, 26, 27] if we enlarge the manifold where $\left\{X^{i}\right\}$ and $\left\{Y_{i}\right\}$ are treated as independent coordinates. Let $\mathrm{W}$ stands for the $2(p+1)$ coordinates $\left\{X^{i}, Y_{i}\right\}$ collectively, then equations (20) and (21) can be expressed as $\mathcal{M} \eta \partial_{\alpha} W=\epsilon_{\alpha \beta} \gamma^{\beta \delta} \partial_{\delta} W$. The evolution equation becomes $\partial_{\alpha}\left(\eta \mathcal{M}^{-1} \gamma^{\alpha \beta} \partial_{\beta} W\right)=0$ which is $O(p+1, p+1)$ invariant.

Now let us focus attention on $\mathcal{L}_{2}$, which can be expressed in the following form after eliminating auxiliary fields $V_{\alpha}^{i}$ in favor of coordinates $Y_{i}$ through (17).

$$
\mathcal{L}_{2}=\frac{1}{2}\left[\gamma^{\alpha \beta} \partial_{\alpha} Y_{i} \partial_{\beta} Y_{j} P^{i j}+\epsilon^{\alpha \beta} \partial_{\alpha} Y_{i} \partial_{\beta} Y_{j} Q^{i j}\right]
$$

The resulting equation motion, in the presence of constant backgrounds $P^{i j}$ and $Q^{i j}$, are

$$
\partial_{\alpha}\left(\gamma^{\alpha \beta} \partial_{\beta} Y_{j} P^{i j}+\epsilon^{\alpha \beta} \partial_{\beta} Y_{j} Q^{i j}\right)=0
$$


with the boundary condition

$$
P^{i j} Y_{j}^{\prime}-Q^{i j} \dot{Y}_{j}=0
$$

We recall that, in the presence of the antisymmetric background $Q^{i j}$, the coordinates $\left\{Y_{i}\right\}$ will exhibit noncommutativity property. In order to demonstrate this attribute, one needs to go over to Euclidean worldsheet description and introduce complex coordinates $(z, \bar{z})$ as mentioned earlier.

Let us now, utilize the relations between $\left(\dot{Y}_{i}, Y_{i}^{\prime}\right)$ and $\left(\dot{X}^{i}, X^{\prime i}\right)$, from (20) and (21), to write the mixed boundary condition (24) in terms of the $\left\{X^{i}\right\}$ coordinates. Thus we arrive at

$$
P^{i j}\left(-G_{j k} \dot{X}^{k}+B_{j k} X^{\prime k}\right)-Q^{i j}\left(-G_{j k} X^{\prime k}+B_{j k} \dot{X}^{k}\right)=0
$$

Now substitute the relations between $P^{i j}, Q^{i j}, G_{i j}$ and $B_{i j}$ using (18) in the above equation which leads to

$$
\dot{X}^{j}=0
$$

We have shown that if one starts from a theory with constant metric and antisymmetric tensor field with mixed boundary conditions (24), so that one has a noncommutative theory; it is possible to go over to a theory described by a set of dual coordinates, with a (corresponding) constant metric, satisfying Dirichlet boundary conditions (26) and consequently, the dual theory is a commutative one.

Next, we address another question in this context. Suppose, we start with a commutative theory such that $G_{i j}$ are constant and $B_{i j}$ are set to zero with Neumann boundary condition i.e. $G_{i j} X^{\prime j}=0$; then, is it possible to generate a B-field and a mixed boundary condition? Our answer is affirmative in this regard as is illustrated by the following example.

Lets us consider infinitesimal $O(p+1, p+1)$ transformation introduced by Maharana and Schwarz [27] where $\Omega$ has the following form

$$
\Omega=\left(\begin{array}{cc}
\mathbf{1}+\alpha & \beta \\
\lambda & \mathbf{1}-\alpha^{T}
\end{array}\right)
$$

Here, $\alpha, \beta$ and $\lambda$ are infinitesimal parameters (actually $(p+1) \times(p+1)$ matrices). The constraint $\beta^{T}=-\beta$ and $\lambda^{T}=-\lambda$ follow from the condition $\Omega \eta \Omega^{T}=\eta$. Let us consider an initial configuration

$$
\mathcal{M}=\left(\begin{array}{cc}
G^{-1} & 0 \\
0 & G
\end{array}\right)
$$

and implement the transformation (27), then we find that

$$
G_{i j} \rightarrow \tilde{G}_{i j}=G_{i j}-G_{i l}\left(\alpha^{T}\right)_{j}^{l}-\alpha_{i}^{l} G_{l j}
$$


We have retained only linear terms in the infinitesimal matrices in the above equation. Note that the pair of 'phase space variables' $\left\{P_{i}, X^{\prime i}\right\}$ will also get transformed according to $(9)$ and $\tilde{X}^{\prime i}=-\lambda^{i j} P_{j}+\left(1+\alpha^{T}\right)_{j}^{i} X^{\prime j}$. Therefore, keeping only the linear terms in infinitesimal parameters, we arrive at the following form of mixed boundary conditions,

$$
-G_{i j} \lambda^{j k} G_{k l} \dot{X}^{l}+\left(G_{i j}-\alpha_{i}^{k} G_{k j}\right) X^{\prime j}=0
$$

using the definition $P_{i}=G_{i j} \dot{X}^{j}$. In (30) we have made a special choice for the infinitesimal parameter $\alpha^{i j}$ is symmetric and is proportional to $\delta^{i j}$.

Notice that we have generated a constant antisymmetric tensor field $B_{i j}=G_{i k} \lambda^{k l} G_{l j}$, through a T-duality transformation by choosing a specific form of the matrix $\Omega, \lambda$ being the infinitesimal matrix valued parameter. As is well known, there are distinctions between a theory where $B=0$ and the one with constant, nonzero B-field for the situation under consideration. Let us introduce the physical vertex operator for gauge field: $V_{A}=\int \xi . \partial X e^{i p . X}$, where $\xi_{i}$ is the polarization vector and for the physical vertex operator the constraints are $\xi \cdot p=0$ and $p^{2}=0$. Recall that all contraction here are done with open string metric $\bar{G}_{i j}$. For the $B=0$ case, when the correlations of vertex operators are computed using (6), there is no $\theta$ dependence; in other words we are dealing with ordinary gauge fields. On the other hand, when Bfield is nonzero, there will be $\theta$ dependent terms if we calculate correlations of gauge field vertex operators. In fact the product of the vertex operators in this case is the * product with the identification of $\theta^{i j}$ as the noncommutative parameter of the $*$ product. Let us recall briefly, the gauge field dynamics from the perspective of $\sigma$-model. The gauge field background term $-\int_{\partial \Sigma} d \tau A_{i} \partial_{\tau} X^{i}$ appearing in (1) is invariant under $\delta_{\psi} A_{i}=\partial_{i} \psi$, and $\psi$ being the gauge parameter. This is valid at the classical level since the gauge variation goes to a total derivative ensuring gauge invariance. However, in the field theoretic frame work, a regularization prescription needs to be adopted while computing the variation. If the Pauli-Villars regularization is chosen the corresponding theory is an ordinary gauge theory whereas for point splitting regularization the gauge transformation required is that of noncommutative theory as has been argued by Seiberg and Witten [11]. We also mention that in case of the Pauli-Villars regularization, when one constructs effective action, its B and F dependence appears in the combination of $B+F$ and the gauge invariance is guaranteed due to the transformation properties: $\delta B=d \Lambda$ and $\delta A=\Lambda, \Lambda$ being a one form. Therefore, the effective action one gets is intimately connected with the choice of regularization prescription in the context of the $\sigma$-model: Pauli-Villars regularization leads to ordinary gauge theory and point splitting to the noncommutative one. Let us adopt the notation $A$ and $\hat{A}$ for the ordinary and noncommutative gauge fields respectively and similarly for the gauge parameters following [11]. The Seiberg-Witten map provides means to express $\hat{A}$ in terms of $A$ and $\hat{\psi}$ in terms of $A$ and $\psi$. If one starts with a theory with 
field strength $\hat{F}$ and noncommutative parameter $\theta$, then the relation is

$$
\hat{F}=\frac{1}{1+F \theta} F
$$

where $F=\hat{F}(\theta=0)$. Therefore, an ordinary gauge field with constant curvature and NS two form field B is equivalent to noncommutative gauge field theory with the appropriate $\theta$-parameter. Thus it follows from (31) that,for $\theta=\frac{1}{B}$

$$
\hat{F}=B \frac{1}{B+F} F
$$

It is obvious from (31) and (32) that $\hat{F}$ will blow up at some points and same argument will go through when we express $\mathrm{F}$ in terms of $\hat{F}$ and $\theta$ by inverting the equation.

In our work, we are able to generate a constant B-field through duality transformations. Therefore, we can construct the Seiberg-Witten map explicitly in terms of the parameter of the noncompact symmetry transformation. The two field strengths are now related by the expression

$$
\hat{F}=G \lambda\left(G \lambda+F G^{-1}\right)^{-1} F
$$

and the matrix multiplications are obvious in the above formula.

We know that noncommutative field theories have the unusual feature that UV and IR cutoffs get interlinked as has been discussed recently [28]. The point is IR diverges appear from the contribution of the nonplanar diagrams when the momenta of external particles go to zero. It is argued that the star product appearing in the action of noncommutative theories are neither maximally Lorentz invariant nor local. The origin of nonlocality can be traced to the fact that in defining the star products one introduces an infinite number of derivatives (note however, that the quadratic part of the action remains same for ordinary product of fields or star products). If we look at two noncommuting spatial coordinates i.e. $\left[X^{i}, X^{j}\right]=1 \theta^{i j}$, it can be argued that a short distance scale in one coordinate, say $X^{i}$ corresponds to a long distance regime in $X^{j}$, since noncommutativity gives rise to a built in uncertainty relation $\Delta X^{i} \Delta X^{j} \geq \frac{1}{2}\left|\theta^{i j}\right|$. Now, for our case same issue can be raised. From string theory point of view, if one considers, scattering of gauge bosons then there will be oneloop open string diagram which one would have to consider. But the same diagram from the closed string point of view is a tree diagram. In fact the relation between noncommutative and ordinary gauge theory processes can be viewed in a more transparent way, if one considers the zero slope limit and then scales metric, $\alpha^{\prime}$ and the B-field appropriately as is explained in [11]. However, we are not in a position to go over to zero slope limit and adopt the scaling of 111 in a straight forward manner, since our B-field is generated by the infinitesimal transformation. Nevertheless, the nonlocality picture should be viewed from the point of view of closed and open string diagrams as mentioned above. 
To summarize, one of our interesting results is that we started with a theory endowed with noncommutative string coordinates since the constant backgrounds $G$ and $\mathrm{B}$ are nonvanishing and the mixed boundary conditions. Then we showed that there exist a set of dual coordinates and backgrounds such that these coordinates satisfy Dirichlet boundary conditions and these dual coordinates belong to a commutative theory. Thus there is an interesting interconnection between a theory which is endowed with the noncommutative and a commutative theories.

We presented another example to show that the infinitesimal noncompact symmetry transformation $\Omega$ generates mixed boundary condition for a special choice of the parameter. We also presented arguments to establish relation between the parameters of the duality transformation and Seiberg-Witten map.

\section{Acknowledgement}

We are thankful to the anonymous referee for several constructive suggestions. We would like to thank the participants of the extended workshop held at MRI in 1999.

\section{References}

1. A. Connes, M. R. Douglas and A. Schwarz, JHEP 9802 (1998) 003,hep-th/971112.

2. M. R. Douglas and C. Hull, JHEP 9802 (1998) 008, hep-th/971165.

3. Y. -K. E. Cheung and M. Krog, Nucl. Phys. B 528 (1998) 185, hep-th/9803031.

4. T. Kawano and K. Okyuyama, Phys. Lett. B433 (1998) 29, hep-th/9803044.

5. C. Hofman and E. Verlinde, JHEP 9812 (1998) 010. hep-th/9810116.

6. N. Seiberg, Talk given at the New Ideas in Particle Physics and Cosmology, Univ. of Pennsylvania, May 19-22, 1999.

7. C.-S. Chu and P.-M. Ho, Nucl. Phys. B550 (1999), hep-th/9812219; Nucl. Phys. B568 (2000) 503.

8. V. Schomerus, JHEP 9906 (1999) 030 hep-th/9903205

9. F. Ardalan H. Arfaei and M. M. Sheikh-Jabbari, Dirac Quantization of Open Strings and Noncommutativity in Branes, hep-th/9906161.

10. N. Ishibashi, A Relation between Commutative and Noncommutative Description of D-branes, hep-th/9909176. 
11. N. Seiberg and E. Witten, String Theory and Noncommutative Geometry, hepth/9908142.

12. J. H. Schwarz, Superstrings and M TheoryDualities, TASI Summer School Lectures, hep-th/9607201.

13. A. Giveon, M. Porrati and E. Rabinovici, Phys. Rep. C244 (1994) 77-202.hepth/9401139

14. A. Sen, An Introduction to Non-perturbative String Theory, hep-th/9802051.

15. J. Maharana, Recent Developments in String Theory, hep-th/9911200.

16. J. Polchinski, TASI Lectures on D-branes, hep-th/9611050.

17. R.Gopakumar, S.Minwalla, J.Maldacena and A.Strominger, hep-th/0005048. and N.Seiberg, L.Susskind and N.Toumbas, hep-th/0005040 for similar considerations.

18. B. Pioline and A. Schwarz, Morita Equivalence and T-duality (or B versus $\theta$ ), hep-th/9908019.

19. M. M. Sheikh-Jabbari, A note on T-duality, Open strings in B-field Backgrounds and Canonical Transformations, hep-th/9911203.

20. J. Dai, R. G. Leigh and J. Polchinski, Mod. Phys. Lett. A4 (1989) 2073.

21. R. Leigh, Mod. Phys. Lett. A4 (1989) 2767.

22. A. Abouelsaood, C. G. Callan, C. R. Nappi and S. A. Yost, Nucl. Phys. B280 (1987) 599.

23. A. Giveon, E. Rabinovici and G. Veneziano, Nucl. Phys. B322 (1989) 167; A. Shapere and F. Wilczek, Nucl. Phys. B320 (1989) 669.

24. K. S. Narain, H. Sarmadi and E. Witten, Nucl. Phys. B279 (1987) 369.

25. M. J. Duff, Nucl. Phys. B335 (1990) 610.

26. J. Maharana, Phys. Lett. B 296 (1992) 65-70.hep-th/9205016

27. J. Maharana and J. H. Schwarz, Nucl. Phys. B390 (1993) 3-32.hep-th/9207016

28. S.Minwalla,M.V.Raamsdonk and N.Seiberg,hep-th/9912072;M.V.Raamsdonk and N.Seiberg,hep-th/0002186 